\title{
The Use of Large-Diameter Braided Stents in TEVAR for a Patient with Complicated Chronic Type B Aortic Dissection
}

\section{Ziheng $\mathrm{Wu}^{1}$, Liang $\mathrm{Xu}^{2}$, Jun $\mathrm{Bai}^{3}$ and Lefeng $\mathrm{Qu}^{3 *}$}

${ }^{1}$ Department of Vascular and Endovascular Surgery, The First Affiliated Hospital, school of Medical, Zhejiang University, Hangzhou, China

${ }^{2}$ Department of Neurosurgery, The Second Affiliated Hospital, School of Medicine, Zhejiang University, Hangzhou, China

${ }^{3}$ Department of Vascular and Endovascular Surgery, Changzheng hospital, The Second Military Medical University, China

"Corresponding author: Lefeng Qu, Department of Vascular and Endovascular Surgery, Changzheng Hospital, The Second Military Medical University, 415 fengyang Rd, Shanghai 200003,China, Tel: 86-21-81886532; Fax: 86-21-81886531; E-mail: qulefengsubmit@163.com

Rec date: May 08, 2015; Acc date: May 29, 2015; Pub date: May 31, 2015

Copyright: ( 2015 Qu L, et al. This is an open-access article distributed under the terms of the Creative Commons Attribution License, which permits unrestricted use, distribution, and reproduction in any medium, provided the original author and source are credited.

\begin{abstract}
Purpose: To describe the use of large-diameter braided stents in thoracic endovascular aortic repair for a patient with complicated chronic type B aortic dissection.

Methods and Results: A 63-year-old male with complicated chronic type B aortic dissection was admitted in our hospital. Computed tomography angiography showed dissection extending from the aortic arch to the left iliac artery and an expanding false lumen in the descending aorta. The corresponding true lumen collapsed. He was successfully treated using a combination of distal large-diameter braided stents and a proximal stent graft. Computed tomography angiography at the 6-month follow-up showed effective remodeling of the true lumen, complete false lumen thrombosis, and shrinkage of the false lumen.
\end{abstract}

Conclusion: The big-diameter braided stents could be safe and effective in endovascular therapy for such patients with complicated chronic type B aortic dissection.

Keywords: Complicated; Chronic type B aortic dissection; Braided stent

\section{Introduction}

For some complicated chronic type B aortic dissection (CCAD) cases, such as true-lumen collapse, aneurysm formation, or large reentry tears, standard endovascular treatment with entry sealing alone is insufficient. Here we present a case of CCAD, whose true lumen in descending thoracic aortic segment was totally collapsed for about 10 years. The true lumen was recanalized first using two large-diameter braided stents. A stent graft was then deployed proximal to seal the entry site. The case which we are reporting is the first case in literature using large-diameter braided stents in the treatment of CCAD.

\section{Case Report}

A 63-year-old male patient with a 10-year history of type B aortic dissection was admitted in our hospital for continuous lumber back pain. CTA showed dissection extending from the aortic arch to the left iliac artery and an expanding false lumen (the biggest diameter of the thoracic dissection aneurysm was $9.5 \mathrm{~cm}$ ) in the descending thoracic aorta. The corresponding true lumen collapsed from the entry tears to the level of the celiac trunk (Figure 1A). The celiac trunk, the superior mesenteric artery, and the right renal artery originated from the false lumen.

The patient was transferred to DSA (digital subtraction angiography) room the next day. Under general anesthesia, a pigtail catheter was advanced from the right femoral artery into the abdominal aorta and an aortogram showed that the true lumen of the

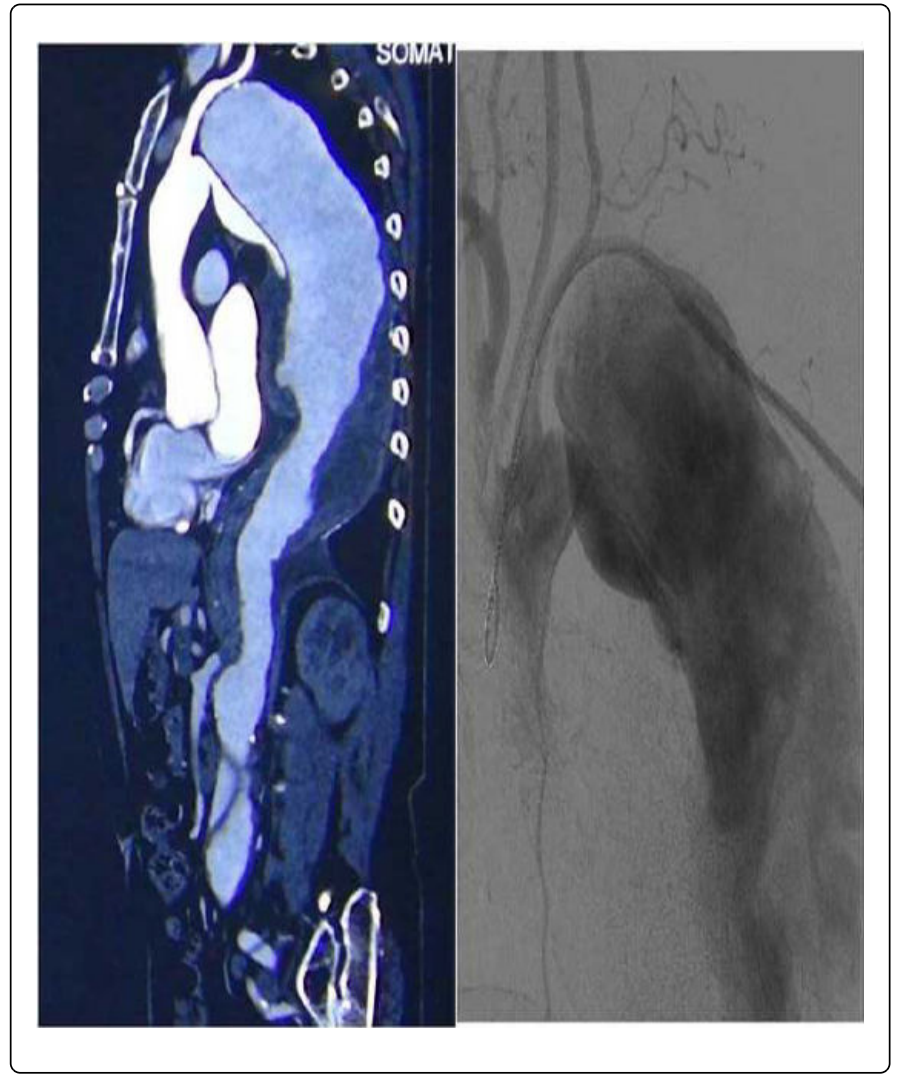




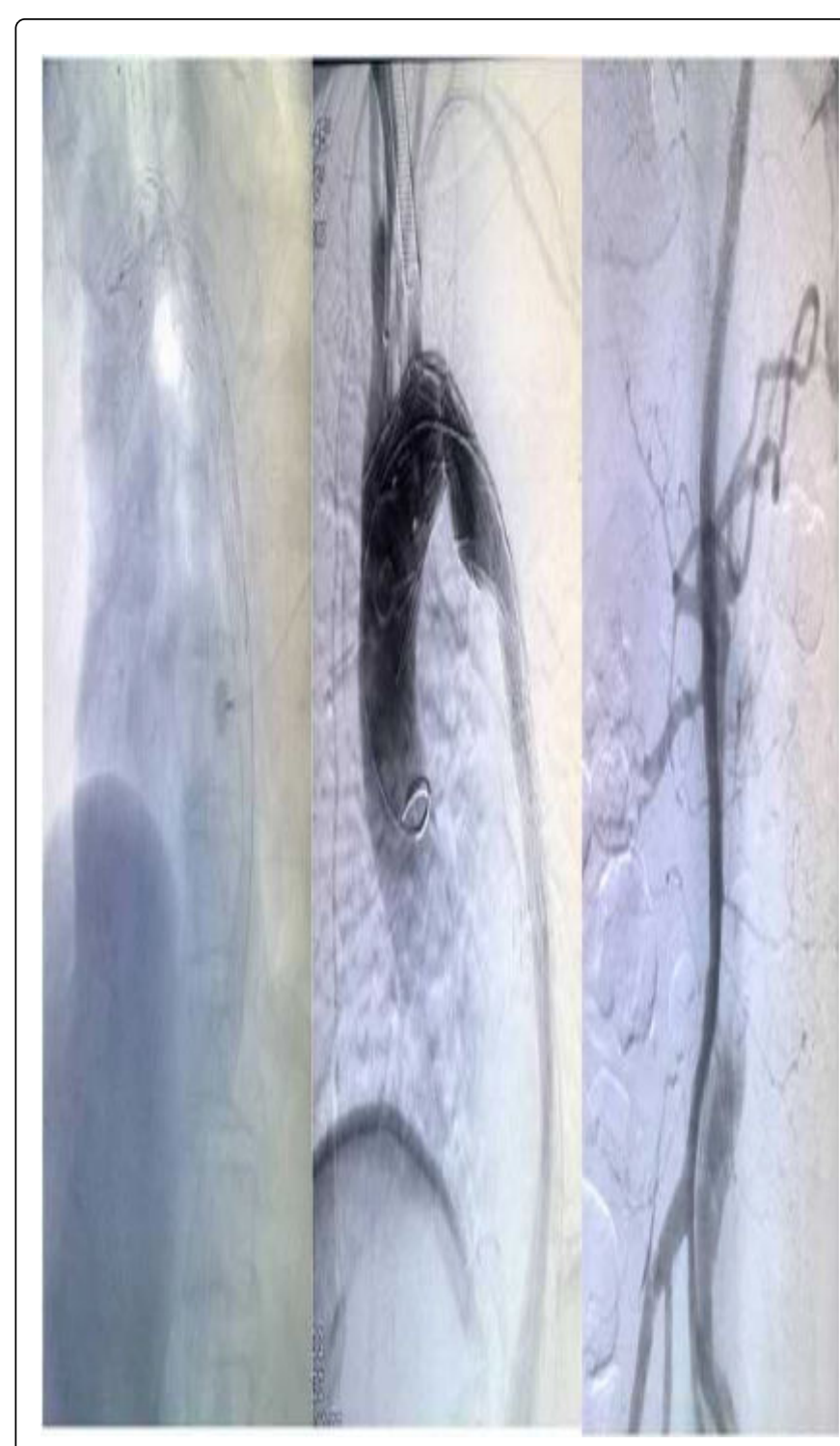

Figure 2(A): Two large-diameter Wallstents ${ }^{\oplus}$ and a stent graft were deployed; (B) Ascending aorta angiography shows that the tears were repaired without endoleaks, the stents were patent, and the left subclavian artery was visualized slowly; (C) Angiography of splanchnic arteries: the superior mesenteric artery, the celiac trunk and the right renal artery were patent.

descending thoracic aorta was totally occluded. The left renal artery could not be visualized. A second aortogram with a catheter from the left brachial artery into the ascending thoracic aorta showed the dissection entry tears were $1.2 \mathrm{~cm}$ distal to the origin of the left subclavian artery and the false lumen of the descending aorta was expanded. The true lumen could not be visualized (Figure 1B). From a right femoral arterial approach, we successfully advanced a guidewire through the true lumen to the ascending of aorta with the assistance of a multipurpose catheter. A $10 \times 40 \mathrm{~mm}$ balloon was used to expand the stenotic segment of the true lumen, followed by the deployment of two Wallstents $^{\oplus}$ (a $24 \times 90 \mathrm{~mm}$ stent in proximal and a $22 \times 90 \mathrm{~mm}$ stent in distal; overlap was about $3 \mathrm{~cm}$ ) in the original totally occluded true lumen. Since the expansion of the proximal portion of the stent was unsatisfactory, a $16 \times 100 \mathrm{~mm}$ balloon was used to dilate. Then a $32 \times 160 \mathrm{~mm}$ stent graft (Hucules T, Microport, SH, China) was deployed in the true lumen, with the proximal end of the stent graft positioned just distal to the origin of the left carotid artery and the distal three segments of the stent graft inside the proximal Wallstents ${ }^{\circ}$ (Figure 2A). A final angiogram was performed to confirm that the tears had been repaired without endoleaks. Angiography of splanchnic arteries showed that the celiac trunk, the superior mesenteric artery, and the right renal artery were also patent (Figure 2B,C).

At discharge, the patient was assigned a daily regimen of aspirin and atorvastatin therapy. The blood pressure was $10 \mathrm{mmHg}$ lower in the left arm but there was no ischemic syndrome. CTA at the 6-month follow-up showed no endoleaks, effective remodeling of true lumen, and completely false lumen thrombosis in the thoracic segment, and shrinkage of the false lumen (Figure 3).

\section{Discussion}

Despite numerous technological advances, the treatment of chronic type B aortic dissections remains controversial and challenging [1]. Nienaber et al.' research showed that in survivors of uncomplicated type B aortic dissection, elective endovascular intervention does not improve 1-year survival rates or lead to a reduction in adverse events, despite favorable aortic remodelling [2]. However, it has been demonstrated that these patients have a high incidence of aortic dissection-related events during long term followup. Evidence suggests that prophylactic intervention for high risk patients may reduce aortic dissection-related events [3]. Currently, most centers advocate endovascular therapy or open surgery for patients with symptoms of continuous pain and evidence of aneurysm formation, malperfusion, rupture or impending rupture [1]. By this criteria, the patient described in this report was truly indicated for intervention, due to his 10-year history of chronic type $\mathrm{B}$ aortic dissections, continuous lumber back pain over a 2 week period, sustained dynamic malperfusion, true lumen collapse and CTA evidence of large aneurysm formation in the descending aorta.

In 2006, Nienaber et al. first introduced the concept of provisional stent graft extension with distal bare metal scaffolding (PETTICOAT) to address the potential complication of sustained distal false lumen flow and pressurization that may occur despite successful sealing of entry tears in patients with complicated type B aortic dissection [4]. Melissano et al. followed with an investigation of the volume changes in aortic true and false lumen after the "PETTICOAT" procedure. Their data showed that use of the PETTICOAT technique may be associated with a significant immediate increase in true lumen volume and resolution of all cases of dynamic malperfusion and true lumen collapse [5]. True lumen collapsed in this patient and standard endovascular therapy with entry tear sealing alone would be insufficient. Like PETTICOAT techniques, our solution was to initiate treatment by deploying two large-diameter Wallstents ${ }^{\circledast}$ in the segment to expand the true lumen. It is important to note that bare metal stents do not influence intercostal artery blood flow, and are thus not likely to cause paraplegia.

Our procedure also differed from the PETTICOAT procedure. In the PETTICOAT, as an adjunctive or staged procedure, a maximum 
Citation: Wu Z, Xu L, Bai J, Qu L (2015) The Use of Large-Diameter Braided Stents in TEVAR for a Patient with Complicated Chronic Type B Aortic Dissection. J Vasc Med Surg S1: S1003. doi:10.4172/2329-6925.S1003

Page 3 of 3

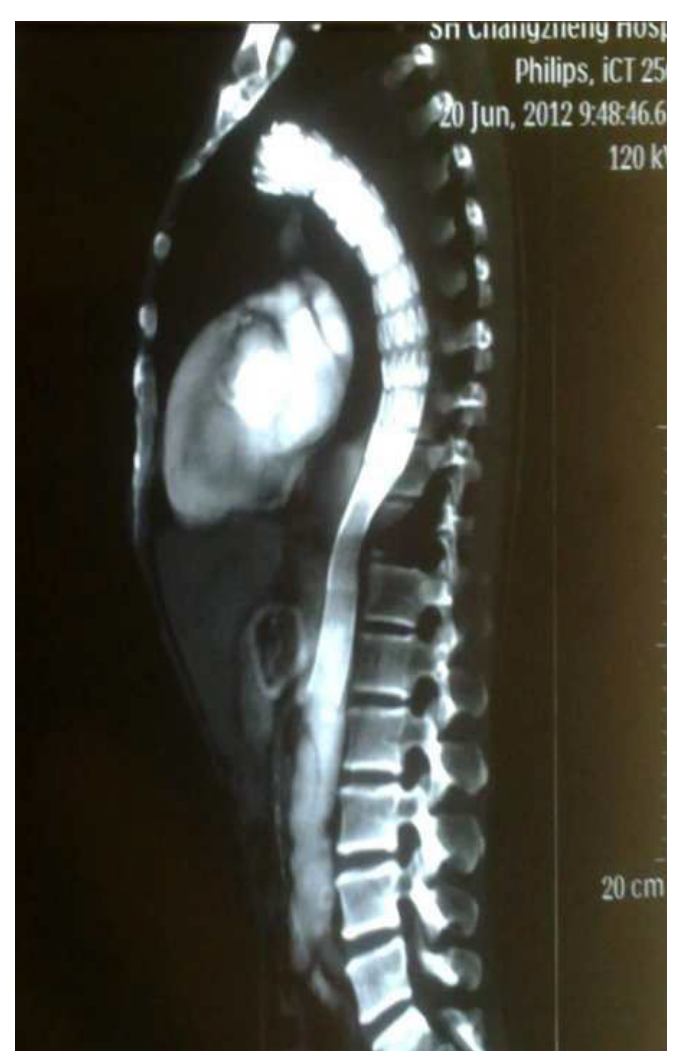

Figure 3: CTA at 6-month follow-up showed no leaks, effective remodeling of the true lumen, completely false lumen thrombosis in th stent implantation.

$2 \mathrm{~mm}$ oversizing laser-cut nitinol stents was placed for distal extension of the previously implanted stent graft. In this case, our opinion was the collapsed descending true lumen had been compressed by the false lumen for ten years and would be better to expand gradually in the re-modeling stage. Therefore we deployed Wallstents ${ }^{\infty}$ in the descending true lumen prior to deployment of the proximal stent graft. So in the early stage, the Wallstents ${ }^{\oplus}$ could prevent redissection by limiting the expansion of the distal end of the stent graft. Besides, in the late remodeling stage, with the false lumen shrinking and true lumen expanding gradually, the Wallstents ${ }^{\oplus}$ could also expand automatically with the true lumen. It should be noted that the Wallstents ${ }^{\oplus}$ would become shorter while expanding gradually in the re-modeling stage and that is why we made enough overlap between the two stents and also enough overlap between the proximal Wallstent ${ }^{\circ}$ and the stent graft.

\section{Conclusion}

From this case, we demonstrated that the big-diameter braided stents could be safe and effective in TEVAR for such patients with CCAD. The re-modeling change should be investigated with longterm follow-up.

\section{References}

1. Simring D, Raja J, Morgan-Rowe L, Haque J, Harris PL, et al. (2011) Placement of a branched stent graft into the false lumen of a chronic type B aortic dissection. J Vasc Surg 54: 1784-7.

2. Nienaber CA, Kische S, Akin I, Rousseau H, Fattori R, et al. (2010) Strategies for subacute/ chronic type B aortic dissection: the Investigation Of Stent Grafts in Patients with type B Aortic Dissection (INSTEAD) trial 1-year outcome. J Thorac Cardiovasc Surg 140: 101-108; discussion: $142-146$.

3. Sueyoshi E, Nagayama H, Hayashida T, Sakamoto I, Uetani M (2014) Fate of aorta and clinical outcomes in patients with chronic type B aortic dissection: over 20-year experience. J Cardiovasc Surg (Torino) 55: 247-255.

4. Nienaber CA, Kische S, Zeller T, Rehders TC, Schneider H, et al. (2006) Provisional extension to induce complete attachment after stent-graft placement in type B aortic dissection: the PETTICOAT concept. J Endovasc Ther 13: 738-746.

5. Melissano G, Bertoglio L, Rinaldi E, Civilini E, Tshomba Y, et al. (2012) Volume changes in aortic true and false lumen after the "PETTICOAT" procedure for type B aortic dissection. J Vasc Surg 55: 641-651.
This article was originally published in a special issue, entitled: "Vascular Medicine and Vascular Surgery", Edited by Dr. Edel P Kavanagh, Galway Clinic and University College Hospital Galway 